\title{
Low-to-Zero-Overhead IRS Reconfiguration: Decoupling Illumination and Channel Estimation
}

This paper was downloaded from TechRxiv (https://www.techrxiv.org).

\section{LICENSE}

CC BY 4.0

SUBMISSION DATE / POSTED DATE

$14-10-2021 / 19-10-2021$

\section{CITATION}

Jamali, Vahid (2021): Low-to-Zero-Overhead IRS Reconfiguration: Decoupling Illumination and Channel Estimation. TechRxiv. Preprint. https://www.techrxiv.org/articles/preprint/Low-to-Zero-

Overhead_IRS_Reconfiguration_Decoupling_Illumination_and_Channel_Estimation/16810420

$\mathrm{DOI}$ 


\title{
Low-to-Zero-Overhead IRS Reconfiguration: Decoupling Illumination and Channel Estimation
}

\author{
Vahid Jamali, Member, IEEE, George C. Alexandropoulos, Senior Member, IEEE, \\ Robert Schober, Fellow, IEEE, and H. Vincent Poor, Life Fellow, IEEE
}

\begin{abstract}
Most algorithms developed so far for the optimization of Intelligent Reflecting Surfaces (IRSs) require knowledge of full Channel State Information (CSI). However, the resulting acquisition overhead constitutes a major bottleneck for the realization of IRS-assisted wireless systems in practice. In contrast, in this paper, focusing on downlink transmissions from a Base Station (BS) to a Mobile User (MU) that is located in a blockage region, we propose to optimize the IRS for illumination of the area centered around the MU. Hence, the proposed design requires the estimation of the MU's position and not the full CSI. For a given IRS phase-shift configuration, the end-to-end BS-IRS-MU channel can then be estimated using conventional channel estimation techniques. The IRS reconfiguration overhead for the proposed scheme depends on the MU mobility as well as how wide the coverage of the IRS illumination is. Therefore, we develop a general IRS phase-shift design, which is valid for both the near- and far-field regimes and features a parameter for tuning the size of the illumination area. Moreover, we study a special case where the IRS illuminates the entire blockage area, which implies that the IRS phase shifts do not change over time leading to zero overhead for IRS reconfiguration.
\end{abstract}

Index Terms-Intelligent reflecting surfaces, channel estimation, blockage area, illumination area, low-overhead design.

\section{INTRODUCTION}

Intelligent Reflecting Surfaces (IRSs) have attracted significant attention as an enabling technology for the realization of smart radio environments in future sixth generation $(6 \mathrm{G})$ wireless systems [1], [2]. However, the envisioned performance gain of IRSs mostly relies on the availability of Channel State Information (CSI), which given the typically large number of reflecting elements, denoted henceforth by $Q$, translates into a huge, often unaffordable, CSI acquisition overhead [3], [4].

Various channel estimation techniques have been proposed in the literature; see [3] for a recent overview. For example, the ON/OFF protocol proposed in [5] comprises $Q$ stages, where in each stage, only one reflecting element is $\mathrm{ON}$ and the corresponding cascaded Base Station (BS)-IRS-Mobile User (MU) channel is estimated. To improve the estimation accuracy, a Discrete Fourier Transform (DFT) based protocol was proposed in [6] which again employs $Q$ stages but, in each stage, all IRS elements are ON and their reflection

V. Jamali and H. Vincent Poor are with the Department of Electrical and Computer Engineering, Princeton University, Princeton, NJ 08544 USA (email: \{jamali, poor\}@ princeton.edu).

G. C. Alexandropoulos is with the Department of Informatics and Telecommunications, National and Kapodistrian University of Athens, Panepistimiopolis Ilissia, 15784 Athens, Greece. (e-mail: alexandg@di.uoa.gr).

R. Schober is with the Institute for Digital Communications at FriedrichAlexander University Erlangen-Nürnberg (FAU), Erlangen, Germany (e-mail: robert.schober@fau.de). coefficients are designed based on one of the columns of the DFT matrix. In [7], the authors proposed an algorithm which estimates the BS-IRS and IRS-MU channels on two different time scales. In particular, they exploited the fact that the BSIRS channel changes much more slowly than the IRS-MU channel, since the IRS and the BS are fixed nodes and the IRS is often deployed to ensure a Line-of-Sight (LoS) BS-IRS link. Nonetheless, the overhead of all of these channel estimation techniques scales with $Q$, which hinders their application for practically large IRSs.

Two categories of IRS channel estimation schemes have been proposed in the literature whose overhead does not scale with $Q$, namely sparsity- and codebook-based schemes [8], [9]. In particular, the sparsity of the wireless channel in the angular domain was exploited in [8] to design a channel estimation algorithm. Therefore, the corresponding overhead scales with the number of dominant propagation paths of the wireless channel. In contrast, in [9], the authors proposed to estimate the end-to-end BS-IRS-MU channel only for a limited number of IRS phase-shift configurations drawn from a codebook. Since the channel estimation overhead scales with the codebook size, an IRS phase-shift profile was developed, which allows the design of small-size phase-shift codebooks.

Regardless of which of the above CSI acquisition schemes is adopted, the common drawback of optimizing the IRS based on CSI is that, since channel gains change quickly, the IRS should be in principle frequently reconfigured (e.g., typical channel coherence times are on the order of milliseconds [10]). In contrast, in this paper, we propose to decouple the IRS reconfiguration from channel estimation. We focus on downlink transmissions from a BS to an MU that is located in a blockage region. The basic idea behind the proposed scheme is that the IRS is configured to illuminate the MU and is only reconfigured if the MU's Quality-of-Service (QoS) requirement cannot be met. Hence, the frequency of IRS reconfiguration does not explicitly depend on the channel coherence time, but on the mobility of the user, the size of the area illuminated by the IRS, and the MU QoS requirement. Once the IRS is configured, it acts as a virtual channel scatterer and the end-to-end BS-IRS-MU channel can be estimated based on conventional channel estimation techniques, and with a frequency that is dictated by the channel coherence time [11]. To facilitate the proposed approach, we develop a general IRS phase-shift design, which unlike those in [9], [12] is valid for both the near- and far-field regimes and features a parameter for tuning the size of the illuminated area. We analyze the corresponding overhead and compare it with that 


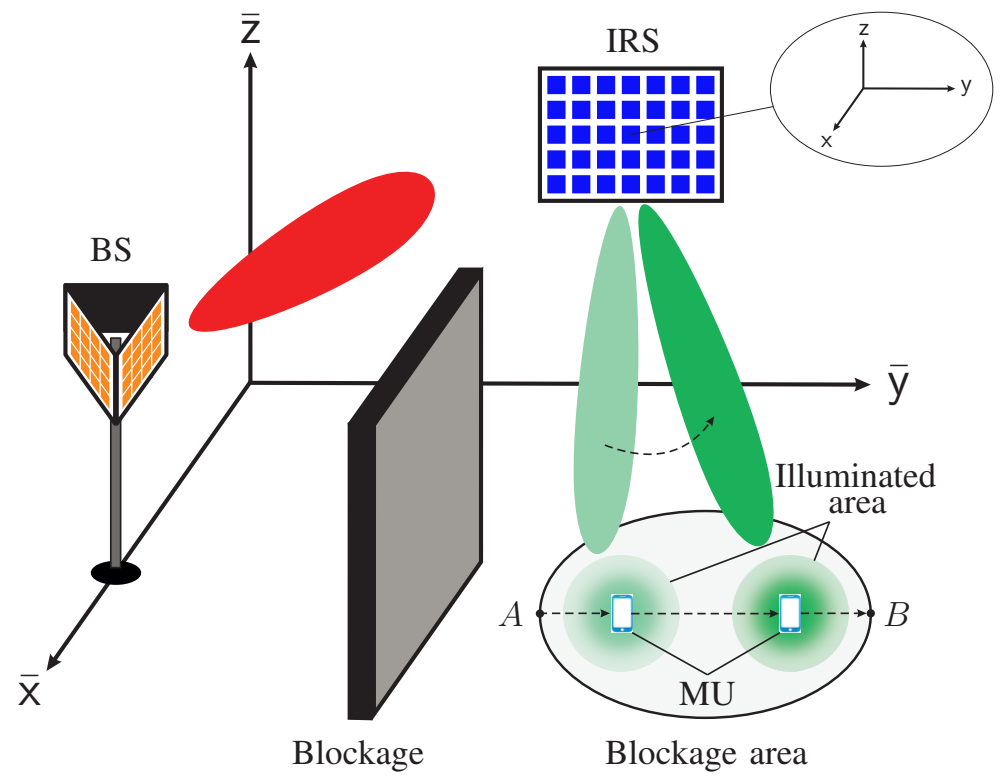

Fig. 1. Schematic illustration of the considered IRS-assisted downlink communication system. Throughout the paper, we mainly use the coordinate system $x y z$ and employ the coordinate system $\bar{x} \bar{y} \bar{z}$ to only represent the positions of the multi-antenna BS, $Q$-element IRS, and single-antenna MU. The generic notations $\mathbf{p}_{i}$ and $\overline{\mathbf{p}}_{i}$ refer to points in the $\mathrm{xyz}$ and $\bar{x} \bar{y} \bar{z}$ systems, respectively.

of the existing schemes. Moreover, we study an interesting special case where the IRS illuminates the entire blockage area, avoiding the need for IRS reconfiguration.

\section{Communication Setup}

In this section, we introduce the considered communication system.

\section{A. System Model}

We consider a downlink system, where a multi-antenna BS wishes to serve a single-antenna MU. We assume that there exists an area where direct coverage is not available due to, e.g., the presence of a blocking building, see Fig. 1. To address this issue, an IRS is deployed such that it has a LoS connection to both the BS and the MU, enabling the realization of a virtual non-LoS link between the BS and the MU. Since the BS and IRS are fixed nodes, we assume that the BS employs a fixed active beamformer directed towards the IRS. Hence, the equivalent baseband signal model is given by [4]

$$
y=\sum_{q=1}^{Q} h_{r, q} \Gamma_{q} h_{i, q} s+n,
$$

where $s, y$, and $n$ denote the BS's data symbol, the MU's received signal, and the additive white Gaussian noise at the MU with zero mean and variance $\sigma_{n}^{2}$, respectively. Moreover, $h_{i, q}$ denotes the effective channel coefficient (including the impact of BS beamforming) between the BS and the $q$-th reflecting element of the IRS, and $h_{r, q}$ denotes the channel coefficient between the $q$-th reflecting element of the IRS and the single MU antenna. Recall that the IRS is placed such that $h_{i, q}$ and $h_{r, q}, \forall q$, have dominant LoS components. Furthermore, $\Gamma_{q} \triangleq \bar{\Gamma} \mathrm{e}^{\mathrm{j} \omega_{q}}$ is the reflection coefficient of the $q$-th IRS reflecting element, where $\bar{\Gamma}$ denotes the normalized element factor and $\omega_{q}$ is the phase shift applied by the $q$ th element. For simplicity, assuming sub-wavelength element size, a constant element factor $\bar{\Gamma}=4 \pi A_{\mathrm{uc}} \lambda^{-2}$ is assumed in this paper, where $A_{\mathrm{uc}}$ is the area of a reflecting element and $\lambda$ is the wavelength. Note that, in general, $\bar{\Gamma}$ may also depend on the incident and reflected angles of the waves, see [4] for details.

\section{B. Scattering Integral}

While the received signal model in (1) is useful for characterizing the system on the small discrete-time scale, it is too general and abstract for an insightful design and performance analysis of the IRS. To cope with this issue, in this paper, we base our design on the LoS links, which for small subwavelength IRS element spacing, can be accurately characterized by the scattering integral [4, Fig. 4]. In particular, using the scalar representation of this integral, the reflected electric field at the MU position $\mathbf{p}_{r}$ can be derived as

$$
E_{r}\left(\mathbf{p}_{r}\right)=\frac{\tau}{\mathrm{j} \lambda} \int_{\mathbf{y}=-\frac{L_{y}}{2}}^{\frac{L_{y}}{2}} \int_{\mathbf{z}=-\frac{L_{z}}{2}}^{\frac{L_{z}}{2}} E_{i} \mathrm{e}^{\mathrm{j} \varphi(\mathbf{p})} \frac{\mathrm{e}^{\mathrm{j} \kappa\left\|\mathbf{p}_{r}-\mathbf{p}\right\|}}{\left\|\mathbf{p}_{r}-\mathbf{p}\right\|} \mathrm{e}^{\mathrm{j} \omega(\mathbf{p})} \mathrm{dyd} \mathbf{z},
$$

where $E_{i}$ and $\varphi(\mathbf{p})$ are the amplitude and phase, respectively, of the incident wave at point $\mathbf{p}=[0, y, z]$ on the IRS, and $\omega(\mathbf{p})$ is the phase shift applied by that point. $L_{\mathrm{y}}$ and $L_{\mathrm{z}}$ denote the IRS dimension along the $y$ - and z-axes, respectively; $\kappa \triangleq \frac{2 \pi}{\lambda}$ is the wave number; and $\tau$ is a constant ensuring the passivity of the IRS [4, Remark 1]. Assuming that $h_{r, q}$ and $h_{i, q}$ comprise only LoS links and the impact of noise $n$ is neglected, the transmit and received symbol powers $P_{\mathrm{tx}} \triangleq|s|^{2}$ and $P_{\mathrm{rx}} \triangleq|y|^{2}$, respectively, defined based on (1), can be obtained from (2) by relating the electric fields and power 


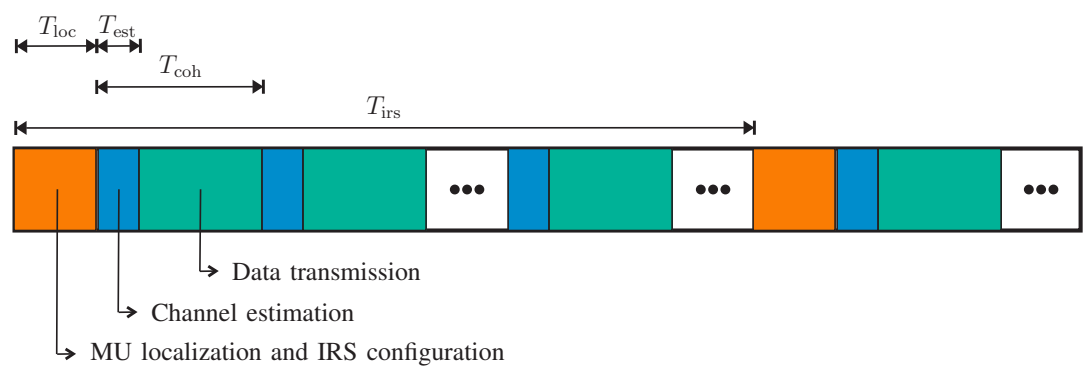

Fig. 2. Block diagram of the proposed IRS-assisted downlink transmission scheme, including the sub-blocks of MU localization, IRS phase-shift design, and pilot-assisted end-to-end channel estimation.

densities in space according to [4, Appendix A]:

$$
P_{\mathrm{rx}}=\frac{\left|E_{r}\right|^{2}}{2 \eta} \frac{D_{\mathrm{rx}} \lambda^{2}}{4 \pi},\left|E_{i}\right|^{2}=2 \eta \frac{P_{\mathrm{tx}} D_{\mathrm{tx}}}{4 \pi d_{i}^{2}} .
$$

The first fraction in the $P_{\mathrm{rx}}$ expression is the received power density, while the second represents the receive antenna aperture with $D_{\mathrm{rx}}$ being the receive antenna directivity. The fraction in the $\left|E_{i}\right|^{2}$ expression is the power density at the IRS with $D_{\mathrm{rx}}$ and $d_{i}$ denoting the transmit antenna directivity and the distance between the BS and the IRS, respectively. Finally, $\eta$ represents the free-space characteristic impedance. We next employ (1) for channel estimation and (2) for the IRS phase-shift design.

\section{User Mobility Model}

Depending on the application of interest, various user mobility models have been proposed in the literature; see [13] for an overview. In this paper, we adopt a simple user mobility model, which is a special case of the random waypoint mobility model [13]. We specifically assume that the MU enters and leaves the area at random points $A$ and $B$, respectively, in Fig. 1, and crosses the area on a straight line with a fixed velocity $v$.

\section{LOW-OVERHEAD IRS RECONFIGURATION}

In this section, we first present the proposed IRS reconfiguration and channel estimation algorithm. Subsequently, we present various IRS phase-shift designs and analyze the overhead of the proposed algorithm.

\section{A. Proposed Algorithm}

The proposed IRS reconfiguration and channel estimation algorithm consists of the following three sub-blocks:

Sub-block 1 (MU Localization): Recall that the IRS is deployed to have LoS connections to both the BS and the MU. Since the IRS and BS are fixed nodes, their relative positions can be estimated once, and then, considered as known. However, the position of the MU, denoted by $\mathbf{p}_{r}$, varies due to its mobility. Various IRS-assisted localization algorithms have been proposed in the literature (see, e.g., [14], [15]). Note that if the MU is in the far field of the IRS, it suffices from this localization sub-block to estimate the field's Angle-of-Departure (AoD) from the IRS to the MU.

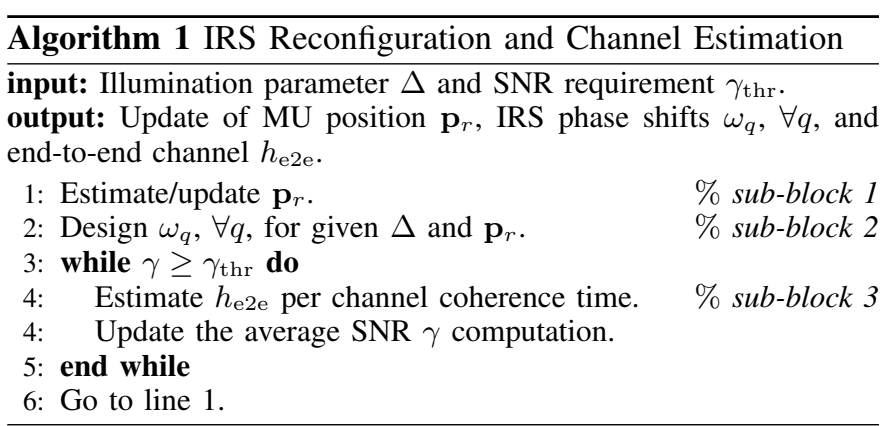

Sub-block 2 (IRS Phase-Shift Design): We assume that the IRS phase-shift design is based only on the MU position or the required field's AoD. However, the IRS radiation pattern can be designed to be narrow [4] or wide [9], [12], depending on the IRS configuration objective. This will be discussed in detail in the following Section III-B.

Sub-block 3 (End-to-End Channel Estimation): Once the IRS is configured, the BS and the MU treat the surface as a part of the end-to-end wireless channel $h_{\mathrm{e} 2 \mathrm{e}} \triangleq \sum_{q=1}^{Q} h_{r, q} \Gamma_{q} h_{i, q}$, which can be estimated using standard (e.g., Least Squares (LS)) channel estimation techniques [11].

Figure 2 illustrates how the above three sub-blocks are employed in the proposed communication protocol, where $T_{\text {loc }}$ denotes the time duration needed by the adopted localization algorithm to estimate/update the MU's position; $T_{\text {est }}$ represents the time duration needed by the adopted channel estimation algorithm to estimate the end-to-end channel; $T_{\text {coh }}$ denotes the channel coherence time; and $T_{\text {irs }}$ is the time duration between two consecutive configurations of the IRS. We assume that the IRS is updated (using sub-blocks 1 and 2) once the current IRS illumination pattern cannot support the MU's QoS. Thereby, we consider that the MU continuously calculates the average Signal-to-Noise Ratio (SNR) $\gamma$ (for which the fading is averaged out, and hence, it is determined by the LoS link), and once it falls below a certain threshold, denoted by $\gamma_{\mathrm{thr}}$, the MU sends a feedback message to the BS to initiate the MU localization and IRS phase-shift update. The proposed IRS configuration and channel estimation algorithm is summarized in Algorithm 1, where $\Delta$ is a parameter that controls the size of the illuminated area, as will be explained in the next subsection. 

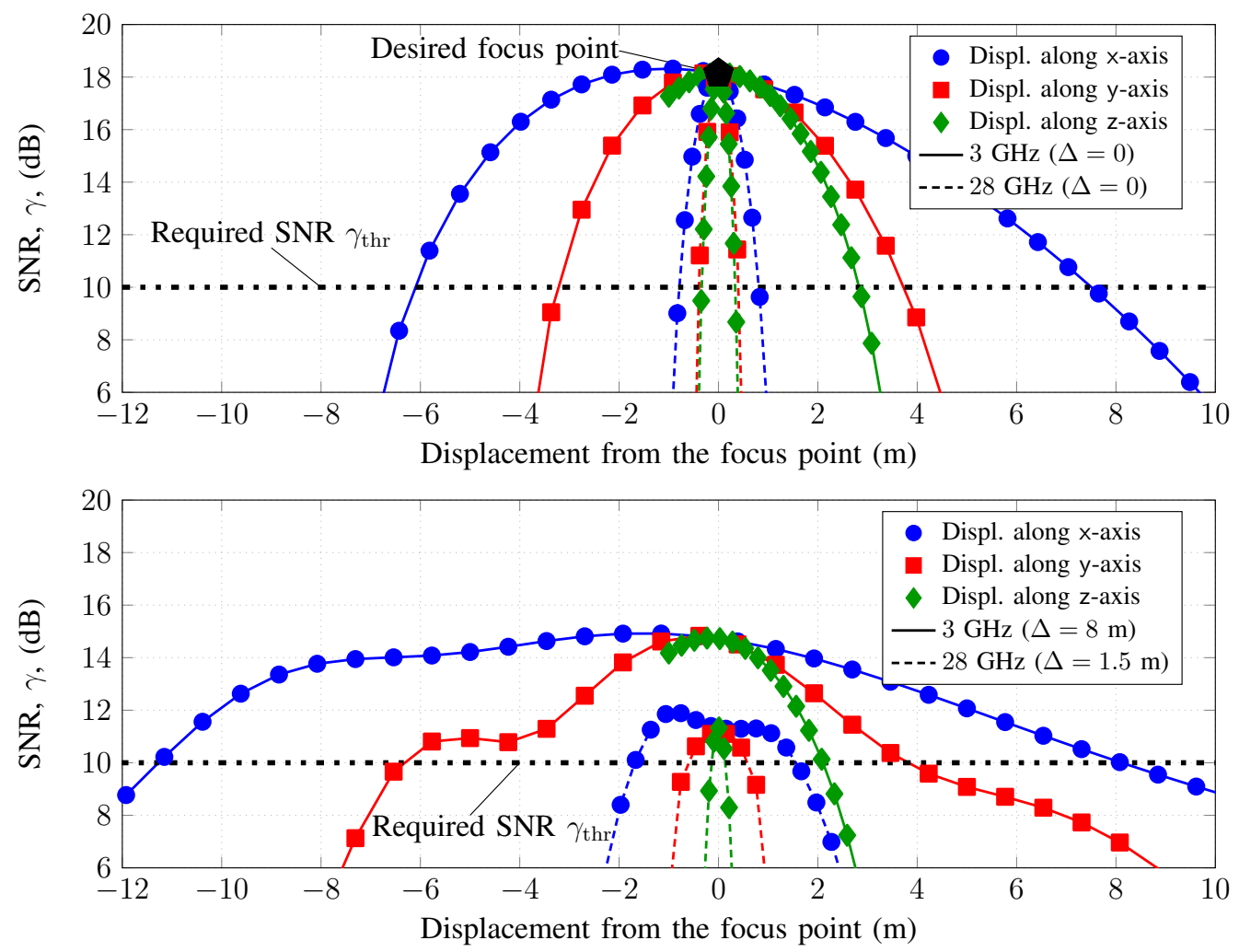

Fig. 3. Received SNR vs. displacement along the $\mathrm{x}-, \mathrm{y}$-, and z-axes around the focus MU position $\overline{\mathbf{p}}_{r}=[20,60,1] \mathrm{m}$ for $L_{\mathrm{y}}=L_{\mathrm{z}}=50 \mathrm{~cm}$, IRS element spacing $d_{\mathrm{y}}=d_{\mathrm{z}}=0.5 \lambda$, BS position $\overline{\mathbf{p}}_{\mathrm{BS}}=[30,0,10] \mathrm{m}$, IRS central point position $\overline{\mathbf{p}}_{\mathrm{IRS}}=[0,50,5] \mathrm{m}, P_{\mathrm{tx}}=10 \mathrm{dBm}, D_{\mathrm{tx}}=12 \mathrm{~dB}, D_{\mathrm{rx}}=0 \mathrm{~dB}$ and $\sigma_{n}^{2}=W N_{0} N_{\mathrm{f}}$ with $N_{0}=-174 \mathrm{dBm} / \mathrm{Hz}, W=20 \mathrm{MHz}$, and $N_{\mathrm{f}}=6 \mathrm{~dB}$. While the BS-IRS and IRS-MU distances are $d_{i}=58 \mathrm{~m}$ and $d_{r}=22 \mathrm{~m}$, respectively, the far-field distance $d_{\mathrm{F}}=8\left(L_{\mathrm{y}}^{2}+L_{\mathrm{z}}^{2}\right) / \lambda$ is $40 \mathrm{~m}$ and $373 \mathrm{~m}$ for $3 \mathrm{GHz}$ and $28 \mathrm{GHz}$, respectively.

\section{B. IRS Phase-Shift Design}

Since various IRS-assisted localization schemes [14], [15] and conventional channel estimation schemes [11] have been proposed in the literature, in this section, we focus our attention on the design and analysis of efficient IRS phaseshift configurations enabling narrow and wide illuminations, respectively.

1) Beam Focusing: In order to maximize the power at the MU position $\mathbf{p}_{r}$ (i.e., maximize $\left|E_{r}\right|$ via (2)), the following phase shift needs to be applied at each point $\mathbf{p}$ on the IRS:

$$
\omega_{\mathrm{F}}\left(\mathbf{p}, \mathbf{p}_{r}\right)=-\kappa\left\|\mathbf{p}_{r}-\mathbf{p}\right\|-\varphi(\mathbf{p}) .
$$

Substituting the above IRS phase shifts in (2) and then in the $P_{\mathrm{rx}}$ expression in (3) yields the maximum average SNR:

$$
\gamma_{\max } \triangleq \frac{P_{\mathrm{rx}, \max }}{\sigma_{n}^{2}}=\frac{P_{\mathrm{tx}} D_{\mathrm{tx}} D_{\mathrm{rx}}}{\sigma_{n}^{2}}\left(\frac{\tau L_{\mathrm{y}} L_{\mathrm{z}}}{4 \pi d_{i} d_{r}}\right)^{2},
$$

where $d_{r}$ denotes the distance between the IRS center and the $\mathrm{MU}$, and we used $\left\|\mathbf{p}_{r}-\mathbf{p}\right\| \approx d_{r}$ to approximate the amplitude $E_{i}$ in (2). The main parameter that determines the overhead of the proposed design is how the SNR decays around the focus point, which is numerically evaluated in the top subfigure in Fig. 3 for an example scenario. As can be observed from this figure for this setup with the SNR requirement of $10 \mathrm{~dB}$, the coverage size along the $x$ - and $y$ - directions around the focus point are $14 \mathrm{~m}$ and $7 \mathrm{~m}(1.6 \mathrm{~m}$ and $0.8 \mathrm{~m})$, respectively, for $3 \mathrm{GHz}(28 \mathrm{GHz})$ carrier frequency. Hence, we can conclude that, for typical walking speeds of approximately $1 \mathrm{~m} / \mathrm{s}$ [13], the IRS update time $T_{\text {irs }}$ is on the order of seconds, which is orders of magnitude larger than typical channel coherence times; those times are on the order of milliseconds [10].

2) Wide Illumination: In order to further reduce the IRS reconfiguration overhead, we propose to widen the reflected beam around the MU's location to increase the area in which the SNR is above the required threshold $\gamma_{\text {thr }}$. Hereby, we propose a systematic approach for IRS phase-shift design for wide illumination. Let $\mathcal{P}_{\text {IRS }}, \mathcal{P}_{\text {blk }}$, and $\mathcal{P}_{\text {ilm }}$ denote the sets of points on the IRS, the blockage area, and the area that we wish to illuminate by the IRS, respectively. The key idea is that focusing on different points $\mathbf{p}_{r}$ in the area that is to be illuminated requires different phase shifts $\omega_{\mathrm{F}}\left(\mathbf{p}, \mathbf{p}_{r}\right)$ at the IRS. Therefore, to illuminate the entire desired area, we have to allocate the IRS area to the area to be illuminated. A general formulation of this problem is a mapping $\mathcal{M}$ from each point $\mathbf{p} \in \mathcal{P}_{\text {IRS }}$ (i.e., the IRS area) to each point $\mathbf{p}_{r} \in \mathcal{P}_{\text {ilm }}$ comprising the targeted illumination area. Given this mapping, the corresponding IRS phase shift for wide illumination, denoted by $\omega_{\mathrm{W}}\left(\mathbf{p}, \mathcal{P}_{\text {ilm }}\right), \forall \mathbf{p} \in \mathcal{P}_{\mathrm{IRS}}$, and $\forall \mathbf{p}_{r}=\mathcal{M}(\mathbf{p}) \in \mathcal{P}_{\text {ilm }}$, can be obtained as a function of $\omega_{\mathrm{F}}(\mathbf{p}, \mathcal{M}(\mathbf{p}))$ in (4), as follows:

$$
\omega_{\mathrm{W}}\left(\mathbf{p}, \mathcal{P}_{\mathrm{ilm}}\right)=\omega_{\mathrm{F}}(\mathbf{p}, \mathcal{M}(\mathbf{p}))-\kappa\left\|\mathcal{M}(\mathbf{p})-\mathbf{p}_{\mathrm{IRS}}\right\| .
$$

The term $\left\|\mathcal{M}(\mathbf{p})-\mathbf{p}_{\text {IRS }}\right\|$ is the reference distance from each point $\mathcal{M}(\mathbf{p})$ to the IRS center, $\mathbf{p}_{\mathrm{IRS}} \triangleq\left[\mathrm{x}_{i}, \mathrm{y}_{i}, \mathrm{z}_{i}\right]$, whose 
contribution is removed from $\omega_{\mathrm{F}}(\mathbf{p}, \mathcal{M}(\mathbf{p}))$. Intuitively, even for focusing, the term $\kappa\left\|\mathcal{M}(\mathbf{p})-\mathbf{p}_{\text {IRS }}\right\|$ only contributes to the signal's phase at the focus point and not to the corresponding power. Since we are mainly interested in the power at the desired observation points within $\mathcal{P}_{\text {ilm }}$, and not in the phase, the contribution of the reference distances, i.e., $\kappa \| \mathcal{M}(\mathbf{p})-$ $\mathbf{p}_{\text {IRS }} \|, \forall \mathcal{M}(\mathbf{p}) \in \mathcal{P}_{\text {ilm }}$, are removed from (6)'s phase-shift design.

Next, we provide two representative examples in order to further elaborate on the general illumination design in (6).

Example 1: Let us assume $L_{\mathrm{y}}=L_{\mathrm{z}}=L$ and a square area $\Delta \times \Delta$ for $\mathcal{P}_{\text {ilm }}$ parallel to the xy plane (i.e., the MU does not change its height). For this case, we propose the following simple mapping from $\mathcal{P}_{\text {IRS }}$ to $\mathcal{P}_{\text {ilm }}$ (needed in (6)):

$$
\mathcal{M}(\mathbf{p}) \triangleq\left[\frac{\Delta}{L} \mathrm{z}+\mathrm{x}_{i}, \frac{\Delta}{L} \mathrm{y}+\mathrm{y}_{i}, \mathrm{z}_{i}\right],
$$

which leads to the following IRS phase-shift design:

$\omega_{\mathrm{W}}\left(\mathbf{p}, \mathcal{P}_{\mathrm{ilm}}\right)=-\kappa\left(\|\mathcal{M}(\mathbf{p})-\mathbf{p}\|-\left\|\mathcal{M}(\mathbf{p})-\mathbf{p}_{\mathrm{IRS}}\right\|\right)-\varphi(\mathbf{p})$.

In other words, each part of the IRS locally focuses on one point in the set $\mathcal{P}_{\text {ilm }}$, and the phase shift across the IRS gradually varies such that all points in $\mathcal{P}_{\text {ilm }}$ are illuminated. The bottom figure of Fig. 3 shows that for carrier frequencies of $3 \mathrm{GHz}$ and $28 \mathrm{GHz}$ using $\Delta=8 \mathrm{~m}$ and $1.5 \mathrm{~m}$, respectively, the areas supporting SNR threshold $\gamma_{\mathrm{thr}}=10 \mathrm{~dB}$ along $(\mathrm{x}, \mathrm{y})$ axis are respectively $(20,10) \mathrm{m}$ and $(3,1.2) \mathrm{m}$, which implies a reduced IRS reconfiguration overhead compared to beam focusing.

Example 2: Suppose that the BS and MU are in the far-field of the IRS. In this case, the phase shift $\omega_{\mathrm{F}}\left(\mathbf{p}, \mathbf{p}_{r}\right)$ given by (4) reduces to the following linear phase shift [4]:

$$
\omega_{\mathrm{F}}\left(\mathbf{p}, \mathbf{\Psi}_{r}\right)=-\kappa\left[A_{\mathrm{y}}\left(\mathbf{\Psi}_{i}, \mathbf{\Psi}_{r}\right) \mathrm{y}+A_{\mathbf{z}}\left(\mathbf{\Psi}_{i}, \mathbf{\Psi}_{r}\right) \mathrm{z}\right],
$$

where $\boldsymbol{\Psi}_{i} \triangleq\left(\theta_{i}, \phi_{i}\right)$ and $\boldsymbol{\Psi}_{r} \triangleq\left(\theta_{r}, \phi_{r}\right)$ denote respectively the incident and reflection angles with $\theta_{u}$ and $\phi_{u}$ for $u \in\{i, r\}$ being the elevation and azimuth angles in the IRS spherical coordinate system, respectively; $A_{t}\left(\boldsymbol{\Psi}_{i}, \boldsymbol{\Psi}_{r}\right) \triangleq A_{t}\left(\boldsymbol{\Psi}_{i}\right)+$ $A_{t}\left(\boldsymbol{\Psi}_{r}\right)$ with $t \in\{\mathrm{y}, \mathrm{z}\} ; A_{\mathrm{y}}\left(\boldsymbol{\Psi}_{u}\right) \triangleq \sin \left(\theta_{u}\right) \sin \left(\phi_{u}\right)$; and $A_{\mathbf{z}}\left(\boldsymbol{\Psi}_{u}\right)=\cos \left(\theta_{u}\right)$. Note that in the far-field regime, the position $\mathbf{p}_{r} \in \mathcal{P}_{\text {blk }}$ can be replaced by the corresponding AoD $\Psi_{r} \in \Psi_{r}$, where $\Psi_{r}$ is the set of possible AoDs. For the ease of presentation, we assume that the range $\boldsymbol{\Psi}_{r} \in \Psi_{r}$ translates into the following intervals for $\alpha_{\mathrm{y}} \triangleq A_{\mathrm{y}}\left(\boldsymbol{\Psi}_{i}, \mathbf{\Psi}_{r}\right) \in$ $\left[A_{\mathrm{y}}^{\min }, A_{\mathrm{y}}^{\max }\right] \triangleq \mathcal{A}_{\mathrm{y}}$ and $\alpha_{\mathrm{z}} \triangleq A_{\mathrm{z}}\left(\boldsymbol{\Psi}_{i}, \boldsymbol{\Psi}_{r}\right) \in\left[A_{\mathrm{z}}^{\min }, A_{\mathrm{z}}^{\max }\right] \triangleq$ $\mathcal{A}_{\mathrm{z}}$. Here, we propose the following mapping from $\mathbf{p} \in \mathcal{P}_{\text {IRS }}$ to $\left(\alpha_{\mathrm{y}}, \alpha_{\mathrm{z}}\right) \in \mathcal{A}_{\mathrm{y}} \times \mathcal{A}_{\mathrm{z}} \triangleq \mathcal{A}_{\mathrm{ilm}}$ (or equivalently to $\boldsymbol{\Psi}_{r} \in \Psi_{r}$ ):

$$
\begin{aligned}
\mathcal{M}(\mathbf{p}) \triangleq & {\left[\frac{A_{\mathrm{y}}^{\max }-A_{\mathrm{y}}^{\min }}{L} \mathrm{y}+\frac{A_{\mathrm{y}}^{\max }+A_{\mathrm{y}}^{\min }}{2},\right.} \\
& \left.\frac{A_{\mathrm{z}}^{\max }-A_{\mathrm{z}}^{\min }}{L} \mathrm{z}+\frac{A_{\mathrm{z}}^{\max }+A_{\mathrm{z}}^{\min }}{2}\right] .
\end{aligned}
$$

Substituting (9) and (10) into the general IRS phase shift in (6), we obtain the illumination of the desired width:

$$
\omega_{\mathrm{W}}\left(\mathbf{p}, \mathcal{A}_{\mathrm{ilm}}\right)=\frac{A_{\mathrm{y}}^{\max }-A_{\mathrm{y}}^{\min }}{L} \mathrm{y}^{2}+\frac{A_{\mathrm{y}}^{\max }+A_{\mathrm{y}}^{\min }}{2} \mathrm{y}
$$

TABLE I

COMPARISON OF THE OVERHEAD OF CSI ACQUISITION AND IRS RECONFIGURATION SCHEMES.

\begin{tabular}{||l|c||}
\hline Schemes & Overhead $(\triangleq \min \{\alpha, 1\})$ \\
\hline $\begin{array}{l}\text { ON/OFF-based [5] } \\
\text { or DFT matrix-based [6] }\end{array}$ & $\alpha=\frac{Q N_{\mathrm{plt}} T_{\mathrm{sym}}}{T_{\mathrm{coh}}}$ \\
\hline $\begin{array}{l}\text { Joint sparsity-based [8] } \\
\text { and two-time scale-based [7] }\end{array}$ & $\alpha=\frac{C N_{\mathrm{pth}} \log \left(N_{\mathrm{grd}}\right) T_{\mathrm{sym}}}{T_{\mathrm{coh}}}$ \\
\hline Codebook-based [9] & $\alpha=\frac{N_{\mathrm{cbk}} N_{\mathrm{plt}} T_{\mathrm{sym}}}{T_{\mathrm{coh}}}$ \\
\hline $\begin{array}{l}\text { Prop. decoupling of illumination } \\
\text { and channel estimation }\end{array}$ & $\alpha=\frac{T_{\mathrm{loc}}+\frac{T_{\mathrm{irs}}-T_{\mathrm{loc}}}{T_{\mathrm{coh}}} N_{\mathrm{plt}} T_{\mathrm{sym}}}{T_{\mathrm{irs}}}$ \\
\hline
\end{tabular}

$$
+\frac{A_{\mathrm{z}}^{\max }-A_{\mathrm{z}}^{\min }}{L} \mathrm{z}^{2}+\frac{A_{\mathrm{z}}^{\max }+A_{\mathrm{z}}^{\min }}{2} \mathrm{z} .
$$

This phase-shift design is similar to the quadratic phase-shift profile proposed in [9] for the realization of small-sized IRS phase-shift codebooks.

3) Full Illumination: Next, we discuss an interesting special case where the IRS illuminates the entire blockage area and hence no IRS reconfiguration is required, i.e., the IRS reconfiguration overhead is zero. This may be achieved, e.g., by choosing a sufficiently large $\Delta$ in the phase-shift design proposed in (8). However, in principle, the possibility of coverage via full illumination depends on several factors such as the BS's transmit power, the IRS phase-shift configuration, the MU's mobility, the required QoS, and the size of the blockage area. It is not straightforward to analytically characterize the exact tradeoff between these parameters for the phase-shift configurations proposed in this paper. Therefore, in Section IV, we numerically evaluate this tradeoff in Fig. 5. Moreover, in the following, we introduce an idealized scenario in order to obtain a first-order insight into this tradeoff.

Let us assume that the blockage area is larger than what can be covered by focusing, as realized with (4). We assume an idealized IRS illumination where the entire power received by the IRS is uniformly distributed across the blockage area. Note that such illumination cannot be realized by an IRS [4], but it provides a performance upper bound for the proposed IRS phase-shift design. Using (3), this leads to the following SNR across the blockage area:

$$
\gamma=\frac{P_{\mathrm{tx}} D_{\mathrm{tx}} D_{\mathrm{rx}}}{\sigma_{n}^{2}}\left(\frac{\lambda}{4 \pi d_{i}}\right)^{2} \frac{L_{\mathrm{y}} L_{\mathrm{z}} A_{\mathrm{x}}\left(\mathbf{\Psi}_{i}\right)}{A_{\mathrm{blk}}}
$$

where $A_{\times}\left(\Psi_{i}\right) \triangleq \sin \left(\theta_{i}\right) \cos \left(\phi_{i}\right)$ and $A_{\text {blk }}$ denotes the size of the blockage area. The above expression can be used to characterize the tradeoff between the BS's transmit power, the IRS size, and the size of the blockage area where the SNR constraint $\gamma \geq \gamma_{\text {thr }}$ holds across the entire illuminated area.

\section{Overhead Comparison}

Table I presents the overhead of the proposed IRS reconfiguration and CSI acquisition scheme and that of several benchmark schemes from the literature for the considered communication system detailed in Section II. Before explaining this table, we would like to acknowledge that the exact overhead of each scheme depends on multiple factors, including the operating SNR as well as the accuracy and tuning 


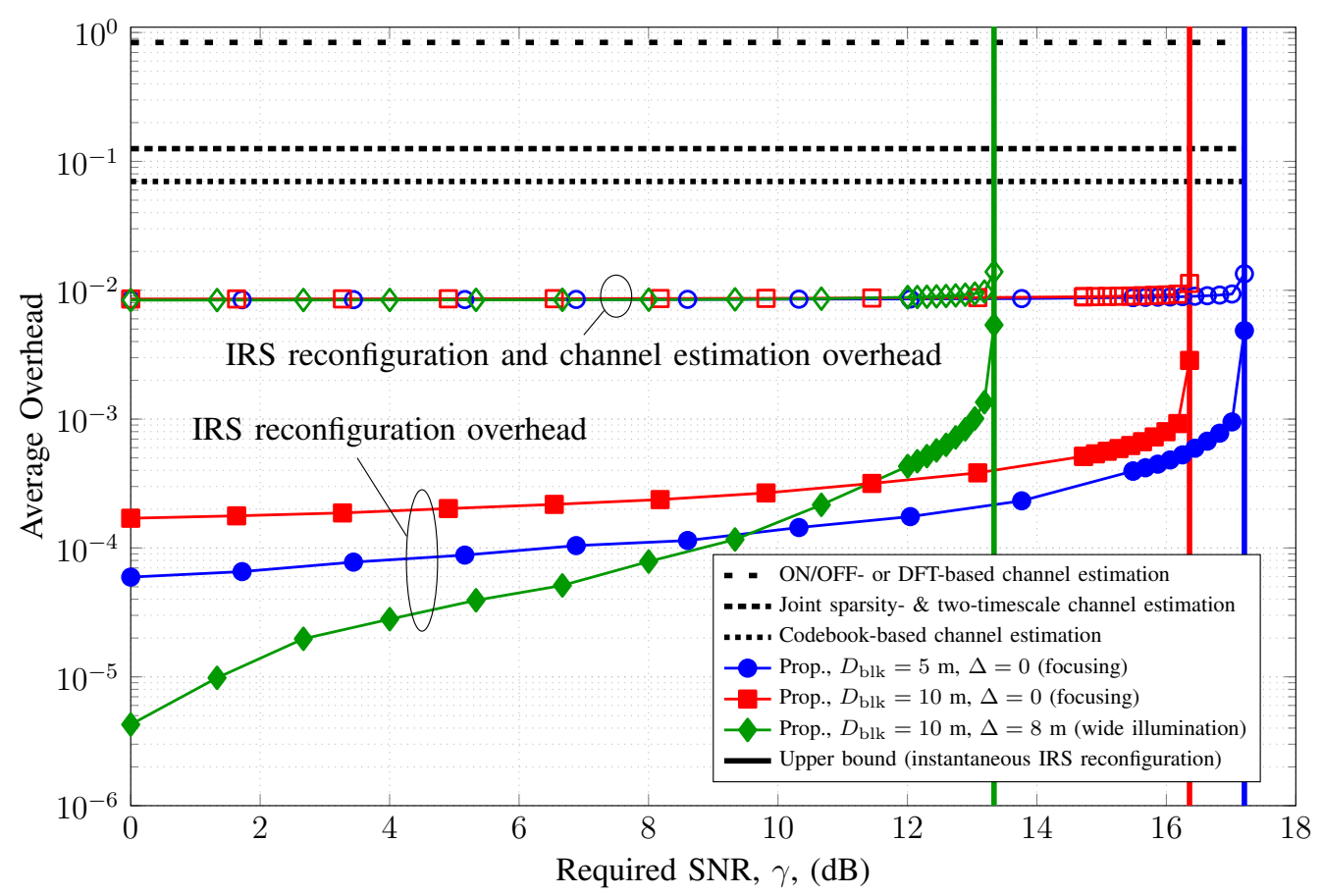

Fig. 4. Average overhead vs. the required SNR for the system parameters used in Fig. 3 and $\overline{\mathbf{p}}_{\text {blk }}=[20,60,1], v=0.75 \mathrm{~m} / \mathrm{s}, N_{\text {plt }}=3, N_{\text {pth }}=5$, $N_{\text {grd }}=20, N_{\text {cdb }}=25, C=1, W_{\text {sub }}=\frac{1}{T_{\text {sym }}}=15 \mathrm{kHz}$, and carrier frequency $3 \mathrm{GHz}$ (i.e., $Q=100$ ).

parameters of the estimation approach, whose quantification is beyond the scope of this paper. Instead, the expressions given in Table I are meant to characterize the scaling behavior of the corresponding overhead in terms of the underlying key parameters.

For the ON/OFF- and DFT matrix-based methods [5], [6], we assume that in each of the $Q$ stages, $N_{\text {plt }}$ pilot symbols of length $T_{\mathrm{sym}}$ are sent. We use the sparsity-based method in [8] jointly with the two timescale-based method in [7], where the overhead of BS-IRS channel estimation is neglected and the number of pilot symbols needed to estimate the IRS-MU channel is assumed to be $C N_{\text {pth }} \log \left(N_{\text {grd }}\right)$ with $N_{\text {pth }}$ and $N_{\text {grd }}$ being the number of paths in the IRS-MU link and the number of discrete grid points, respectively, and $C$ is a constant that depends on the adopted compressed sensing algorithm. The codebook size for codebook-based channel estimation is denoted by $N_{\text {cbk }}$. For the proposed scheme, the value of $T_{\text {loc }}$ in general depends on the adopted localization scheme. However, if sparsity-based localization is adopted and the MU position is assumed to be along the dominant path, the number of required pilot symbols, and hence, $T_{\text {loc }}$ is upper bounded by $C N_{\text {pth }} \log \left(N_{\text {grd }}\right)$ [8].

\section{Simulation Results}

The communication setup considered in this section is schematically illustrated in Fig. 1 and the values of the system parameters are identical to those used for Fig. 3 unless otherwise stated. The channel coherence time is computed based on [10, Eq. (8)] and is approximately $24 \mathrm{~ms}$ at $3 \mathrm{GHz}$ and $v=0.75 \mathrm{~m} / \mathrm{s}$. The blockage area is a circle with center $\overline{\mathbf{p}}_{\mathrm{blk}}$ and diameter $D_{\mathrm{blk}}$. The illumination scheme in (8) is adopted which reduces to the near-field focusing in (4) for $\Delta=0$.

In Fig. 4, we plot the overhead of the proposed IRS reconfiguration and channel estimation scheme as a function of the required SNR. Moreover, we include the channel estimation overhead for the different benchmark schemes given in Table I for comparison. The vertical lines in Fig. 4 represent the maximum SNR that is achievable for all points within the blockage area and can be attained only via instantaneous IRS reconfiguration. This figure suggests that unless the required SNR is extremely close the maximum achievable SNR, the IRS reconfiguration overhead is orders of magnitude less than the channel estimation overhead. In other words, at the cost of a negligible overhead, the proposed scheme can support SNRs which are quite close (e.g., $1 \mathrm{~dB}$ ) to the upper bound. Moreover, we observe from Fig. 4 that compared to near-field focusing $(\Delta=0)$, wide illumination $(\Delta=8 \mathrm{~m})$ yields a lower IRS reconfiguration overhead for low required SNR values.

In Fig. 5, we plot the minimum transmit power needed to support $\gamma_{\mathrm{thr}}=10 \mathrm{~dB}$ via full illumination (i.e., zero IRS reconfiguration overhead) as a function of the blockage area diameter. As can be seen from this figure, for near-field focusing, the transmit power has to significantly increase as the blockage area size increases. In contrast, wider illumination demands a smaller transmit power for larger blockage areas. An unattainable lower bound on the transmit power obtained from (12) and a lower bound that is achievable by instantaneous focusing (incurring overhead) are also plotted for comparison.

\section{REFERENCES}

[1] X. Yu, V. Jamali, D. Xu, D. W. K. Ng, and R. Schober, "Smart and reconfigurable wireless communications: From IRS modeling to 


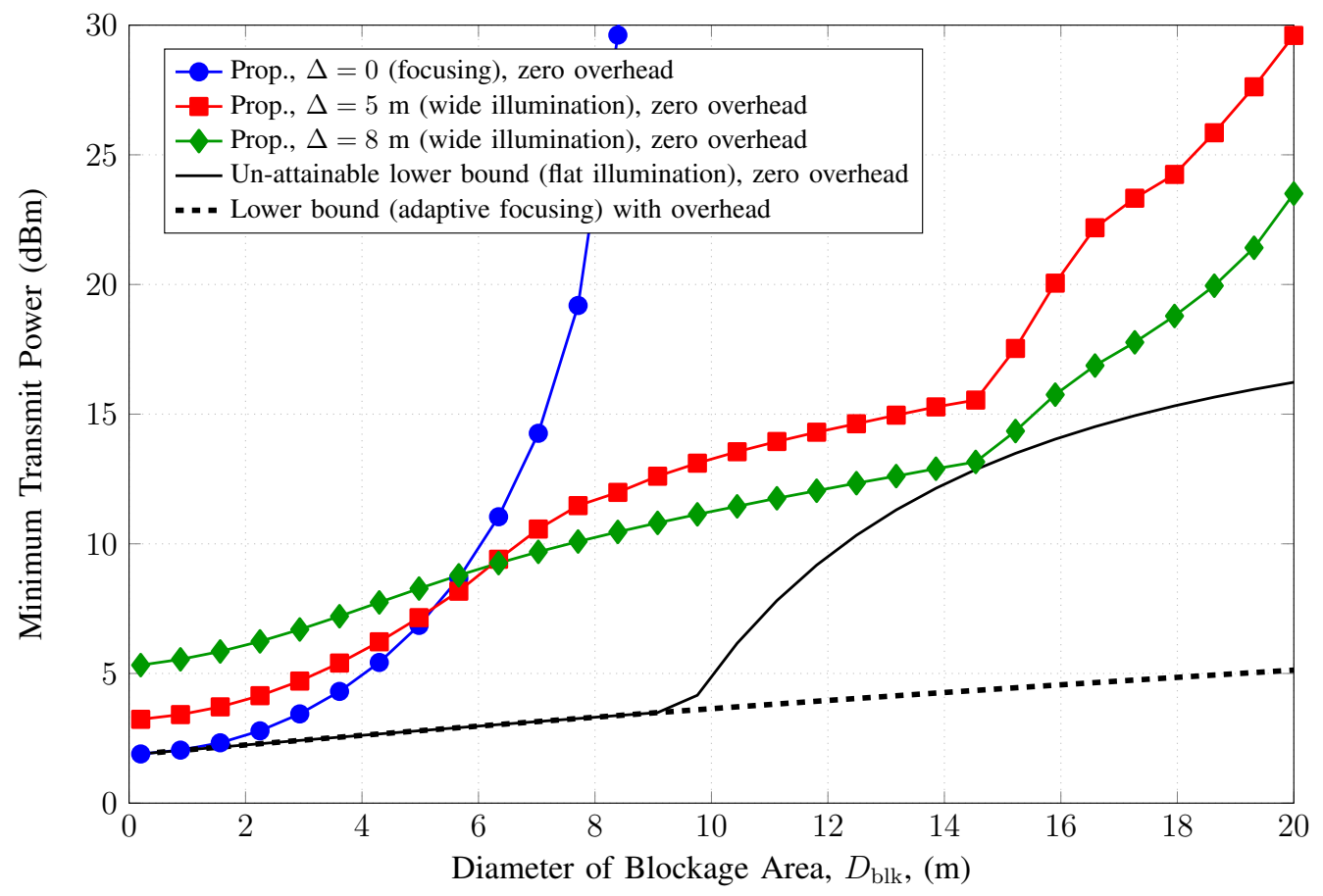

Fig. 5. Minimum transmit power vs. the diameter of the blockage area for the system parameters used in Fig. 3 and $\overline{\mathbf{p}}_{\text {blk }}=[20,60,1]$, required SNR $10 \mathrm{~dB}$, and carrier frequency $3 \mathrm{GHz}$ (i.e., $Q=100$ ).

algorithm design," IEEE Wireless Commun. Mag., to appear, 2021.

[2] E. Calvanese Strinati, G. C. Alexandropoulos et al., "Reconfigurable, intelligent, and sustainable wireless environments for $6 \mathrm{G}$ smart connectivity," IEEE Commun. Mag., to appear, 2021

[3] L. Wei, C. Huang, G. C. Alexandropoulos, C. Yuen, Z. Zhang, and M. Debbah, "Channel estimation for RIS-empowered multi-user MISO wireless communications," IEEE Trans. Commun., vol. 69, no. 6, pp. 4144-4157, Jun. 2021.

[4] M. Najafi, V. Jamali, R. Schober, and H. V. Poor, "Physics-based modeling and scalable optimization of large intelligent reflecting surfaces," IEEE Trans. Commun., vol. 69, no. 4, pp. 2673-2691, Apr. 2021.

[5] D. Mishra and H. Johansson, "Channel estimation and low-complexity beamforming design for passive intelligent surface assisted MISO wireless energy transfer," in Proc. IEEE ICASSP, 2019, pp. 4659-4663.

[6] H. Alwazani, A. Kammoun, A. Chaaban, M. Debbah, and M.-S. Alouini, "Intelligent reflecting surface-assisted multi-user MISO communication: Channel estimation and beamforming design," IEEE Open J. Commun. Society, vol. 1, pp. 661-680, May 2020.

[7] C. Hu, L. Dai, S. Han, and X. Wang, "Two-timescale channel estimation for reconfigurable intelligent surface aided wireless communications," IEEE Trans. Commun., 2021.

[8] P. Wang, J. Fang, H. Duan, and H. Li, "Compressed channel estimation

[14] H. Wymeersch, J. He, B. Denis, A. Clemente, and M. Juntti, "Radio localization and mapping with reconfigurable intelligent surfaces: Chal- for intelligent reflecting surface-assisted millimeter wave systems," IEEE Sig. Process. Lett., vol. 27, pp. 905-909, 2020.

[9] V. Jamali, M. Najafi, R. Schober, and H. V. Poor, "Power efficiency, overhead, and complexity tradeoff of IRS codebook design-Quadratic phase-shift profile," IEEE Commun. Lett., vol. 25, no. 6, pp. 2048-2052, Jun. 2021.

[10] R. P. Torres and J. R. Pérez, "A lower bound for the coherence block length in mobile radio channels," Electronics, vol. 10, no. 4, p. 398, 2021.

[11] L. Liu and W. Yu, "Massive connectivity with massive MIMO-Part I: Device activity detection and channel estimation," vol. 66, no. 11, pp. 2933-2946, Jun. 2018

[12] F. Laue, V. Jamali, and R. Schober, "IRS-assisted active device detection," in Proc. IEEE SPAWC, 2021, pp. 1-5.

[13] Q. Zheng, X. Hong, and S. Ray, "Recent advances in mobility modeling for mobile ad hoc network research," in Proc. 42nd Annual Southeast Regional Conf., 2004, pp. 70-75.

lenges, opportunities, and research directions," IEEE Veh. Technol. Mag., vol. 15, no. 4, pp. 52-61, Dec. 2020.

[15] Z. Abu-Shaban, K. Keykhosravi, M. F. Keskin, G. C. Alexandropoulos, G. Seco-Granados, and H. Wymeersch, "Near-field localization with a reconfigurable intelligent surface acting as lens," in Proc. IEEE ICC, 2021, pp. 1-6. 